\title{
Prognostic value of TP53 expression and MGMT methylation in glioblastoma patients treated with temozolomide combined with other chemotherapies
}

\author{
Maher Kurdi ${ }^{1}$ (]) Nadeem Shafique Butt ${ }^{2} \cdot$ Saleh Baeesa ${ }^{3}$ (1) $\cdot$ Badrah Alghamdi $^{4}$ 'Yazid Maghrabi ${ }^{5} \cdot$ Anas Bardeesi $^{5}$. \\ Rothaina Saeedi ${ }^{3}$ - Ashraf Dallol ${ }^{6}$. Fawaz Mohamed ${ }^{1}$ - Mohammed O. Bari ${ }^{7}$. Alaa Samkari ${ }^{8}$. Ahmed I. Lary ${ }^{9}$. \\ Shadi Alkhayyat ${ }^{10}$
}

Received: 2 February 2021 / Accepted: 18 February 2021 / Published online: 4 March 2021

(c) The Author(s) 2021

\begin{abstract}
Objective To assess the recurrence interval and predictive significance of TP53 expression and O6-methylguanine-DNA methyltransferase (MGMT) promoter methylation in glioblastomas treated with radiotherapy and combined chemotherapies, including temozolomide, lomustine, procarbazine and bevacizumab.

Method We reviewed the clinical outcomes of 52 totally resected glioblastoma patients, who received conventional radiotherapy and temozolomide with other chemotherapeutic agents. Correlation of TP53 expression and MGMT promotor methylation with recurrence interval was analyzed using Kaplan Meier estimates.

Results No significant association was found between MGMT promotor methylation and TP53 expression in glioblastomas $(P$-value $=0.158)$. Patients with non-methylated $M G M T$ who received temozolomide chemotherapy with other chemotherapeutic agents showed significantly later recurrence $(P$-value $=0.007)$ compared with patients with non-methylated $M G M T$ who received temozolomide alone. No significant difference was found in recurrence interval among glioblastoma patients with methylated MGMT who received temozolomide alone or with other chemotherapies $(P$-value $=0.667)$. Moreover, patients with non-TP53-expressing tumors who received temozolomide with other chemotherapies had significantly later recurrence $(P$-value $=0.04)$ compared with patients who received temozolomide alone.

Conclusion Totally resected glioblastoma patients, with non-methylated MGMT or non-TP53-expressing tumors treated with radiotherapy and combined chemotherapies had a reduced chance of tumor recurrence and a more favorable outcome. Furthermore, both MGMT and TP53 are independent prognostic factors for glioblastoma.
\end{abstract}

Keywords Glioblastoma $\cdot M G M T$ promotor methylation $\cdot$ TP53 mutation $\cdot$ Temozolomide

Maher Kurdi

Ahkurdi@kau.edu.sa

1 Department of Pathology, Faculty of Medicine in Rabigh, King Abdulaziz University, Jeddah, Kingdom of Saudi Arabia

2 Department of Family and Community Medicine, Faculty of Medicine in Rabigh, King Abdulaziz University, Jeddah, Kingdom of Saudi Arabia

3 Division of Neurosurgery, Faculty of Medicine, King Abdulaziz University, Jeddah, Kingdom of Saudi Arabia

4 Department of Physiology, Faculty of Medicine, King Abdulaziz University, Jeddah, Kingdom of Saudi Arabia

5 Department of Neuroscience, King Faisal Specialist Hospital and Research Center, Jeddah, Kingdom of Saudi Arabia
6 Center of Excellence in Genomic Medicine Research, Faculty of Applied Medical Science, King Abdulaziz University, Jeddah, Kingdom of Saudi Arabia

7 Department of Pathology, King Abdulaziz University Hospital, Jeddah, Kingdom of Saudi Arabia

8 Department of Pathology and Laboratory Medicine, King Saud Bin Abdulaziz University for Health Science, Jeddah, Kingdom of Saudi Arabia

9 Section of Neurosurgery, Department of Surgery, King Abdulaziz Medical City, Jeddah, Kingdom of Saudi Arabia

10 Division of Oncology, Faculty of Medicine, King Abdulaziz University, Jeddah, Kingdom of Saudi Arabia 


\section{Introduction}

Glioblastoma is the most common primary brain tumor in adults. The standard treatment includes surgical resection followed by radiotherapy (RT) and chemotherapy, mostly with the alkylating agent, temozolomide (TMZ). Despite these regimens, outcomes remain poor [1]. Although RT with TMZ is the first option for newly diagnosed glioblastoma patients, chemotherapy resistance is common. Several parameters are used to predict the prognosis of patients with glioblastoma, including methylation of the O6-methylguanine-DNA methyltransferase gene (MGMT) and mutation of the tumor suppressor gene, Tumor Protein 53 (TP53) [2,3]. The regulation of MGMT is complex and involves TP53, which is required for induction and also for down-regulation to basal levels [4]. Although MGMT methylation is more frequent in tumors expressing mutant $T P 53$, the association between these two factors is uncertain. Hence, high levels of MGMT activity with low TP53 expression are associated with a high TMZ resistance rate. Not all glioblastoma patients with $M G M T$ promoter methylation respond to alkylating agents, and even those who respond eventually experience relapse [5, 6]. MGMT promoter methylation is associated with improved overall survival in patients treated with RT plus temozolomide but not in patients initially treated with RT alone. Indeed, tumor progression-free survival of glioblastoma patients with MGMT methylation is better than that of patients with non-methylated glioblastoma. These findings do not correlate with TP53 expression or TP53 mutation status. Different strategies have been applied to overcome MGMT-mediated chemoresistance but none of them have significantly improved tumor progression-free survival.

Here, we tested 52 totally resected glioblastoma patients for $M G M T$-methylation and TP53 expression. We then assessed the relationship between $M G M T$ promoter methylation and TP53 mutation with recurrence status in glioblastoma patients receiving single or combined chemotherapies. This is the first study of this association in Saudi Arabia.

\section{Material and methods}

\section{Patient stratification}

The study included 52 patients with primary glioblastomas who underwent total surgical resection at different medical institutions in Saudi Arabia between 2013 and 2019. The study was approved by the National biomedical Ethics Committee of King Abdulaziz University (HA-02-J-008).
Histological diagnoses were made according to the World Health Organization (WHO) classification 2016 of CNS tumours. Clinical data, including patients' age at diagnosis, sex, post-operative radiotherapy and chemotherapies, were taken from hospital reports. None of the patients had received preoperative radiation or chemotherapy. Results of MGMT promotor methylation were available for 36 patients from hospital records. The remaining 16 patients involved in this study underwent MGMT methylation status testing using tissue blocks. In total, 54 patients received a standard protocol of radiotherapy (RT) and chemotherapy after surgery while four patients received only radiotherapy. Three patients did not receive any kind of treatment after surgery because of poor health status. All patients who received chemoradiotherapy were stratified into two groups: Group (a) received temozolomide (TMZ) alone $(n=29)$, and Group (b) received TMZ plus other chemotherapeutic agents $(n=16)$. The additional chemotherapeutic agents were a chloroethylating agent (lomustine) with or without procarbazine and bevacizumab. Standard RT was given as a total dose of $60 \mathrm{~Gy}$ and the TMZ was given at $150-200 \mathrm{mg} / \mathrm{m}^{2}$ for 5 days every 28 days for $6-12$ cycles. All patients enrolled in this analysis have unfortunately died.

\section{Tumor samples}

Archival routinely formalin-fixed and paraffin-embedded (FFPE) tumor tissues were collected from 52 patients of Arabic descent, who were histologically diagnosed with glioblastoma. Hematoxylin and eosin (H\&E)-stained sections were re-examined by a certified neuropathologist (MK) to ensure that the histopathological diagnosis was made based on the WHO's classification. One unstained positivecharged slide from each of the 52 FFPE tissue blocks was prepared for TP53 immunostaining. Five $6-\mu$ FFPE sections were obtained from each sample for $M G M T$ methylation sequencing.

\section{Immunohistochemistry (IHC) for TP53}

An anti-TP53 antibody was used to qualitatively identify wild-type and mutant TP53 in FFPE sections using an automated slide stainer. The IHC assay using a mouse monoclonal antibody (IgG1, kappa), directed against human TP53 (DO-7), was performed with the ultraView DAB detection Kit from Ventana on a BenchMark XT automated staining system. A protocol was established so that the entire assay procedure consisted of deparaffinization with EZ Prep at $75^{\circ} \mathrm{C}$, heat pre-treatment in Cell Conditioning medium $(\mathrm{Ag}$ unmasking) ( $\mathrm{CC} 1$; Ventana) for $60 \mathrm{~min}$ and then primary incubation for 16 min at $37{ }^{\circ} \mathrm{C}$. The slides were counterstained with Hematoxylin II for 16 min and bluing reagent 
was used for $16 \mathrm{~min}$. After that, the slides were removed from the slide stainer and then immersed into successive alcohol buffers at different concentrations for $3 \mathrm{~min}$. Sections in which $>10 \%$ of tumor cells were positively stained were defined as "Expressed TP53" and thus mutated (TP53-mt).

\section{DNA extraction and MGMT methylation sequencing}

Thirty-six samples had been previously tested for $M G M T$ methylation using a pyrosequencing method and the results were obtained from hospital records. For the remaining 16 samples, we used qualitative methylation-specific PCR (MSP) to detect MGMT methylation status. The MSP assay detects $\mathrm{CpG}$ island methylation with high sensitivity and specificity. Samples in which a methylated sequence was amplified were scored as methylation positive. The percentage of methylated amplicons detected in an unmethylated control was defined as the cut-off value to separate unmethylated from methylated glioblastomas.

H\&E-stained sections from 16 FFPE tissue blocks were examined by a neuropathologist (MK) to select regions from which DNA could be extracted. DNA was isolated by standard procedures from selected tissue fragments containing a high percentage of tumor cells. DNA extraction was performed using the QIAamp DNA FFPE tissue kit according to the manufacturer's instructions. DNA quantity and quality were determined using a NanoDrop spectrophotometer at A260/A280 and A260/A230. The concentration of DNA samples was normalized to $50 \mathrm{ng}$ and bisulfite-converted using the EpiTect Bisulfite Kit (Qiagen) according to the manufacturer's instructions. Qualitative detection of $M G M T$ methylation was performed using MSP as described previously with modifications [7]. The forward and reverse primers targeting methylated and unmethylated exon 1 of the human MGMT gene are listed in Table 1 and correspond to those described by Esteller et al. [7]. The PCR Kit used was HotStarTaq plus DNA polymerase (Qiagen). Thermal cycling on a Veriti thermal cycler (Thermo Fisher) included an initial step at $95^{\circ} \mathrm{C}$ for 2 min followed by 40 cycles of $30 \mathrm{~s}$ at $94{ }^{\circ} \mathrm{C}, 30 \mathrm{~s}$ at $52{ }^{\circ} \mathrm{C}$, and $30 \mathrm{~s}$ at $72{ }^{\circ} \mathrm{C}$ for $10 \mathrm{~min}$. In vitro methylated and non-methylated control DNA (Qiagen) was used in every run. The PCR products were visualized on $8 \%$ non-denaturing polyacrylamide gels and stained with ethidium bromide. Samples having only methylated PCR products and samples having both methylated and non-methylated PCR products were both scored as methylation positive.

\section{Statistical methods}

Data are described as frequencies and percentages. Recurrence interval (RI) was calculated from the time of starting adjuvant therapy after surgical resection until the first day of tumor recurrence. Chi-Square, Fisher's Exact and Mantel-Haenszel Chi-Square tests were used to explore the association of recurrence status, MGMT promotor methylation status, and TP53 expression with various study factors. Kaplan Meier curves and log-rank tests were used to compare the distribution of recurrence time. All statistical analyses were performed using IBM SPSS1 ver. 24 statistical software (IBM Corp., Armonk, NY).

\section{Results}

\section{Descriptive analysis}

Of the 52 enrolled patients, 21 were less than 50 years old, and 31 were aged 50 or over. All patients were with Arabic descent. The predominant tumor location was the frontal lobe $(n=20,38.5 \%)$ followed by temporal and parietal areas $(n=28)$. Rare locations included the cerebellum and occipital lobe. There were equal numbers of glioblastoma cases with $M G M T$-methylation $(\mathrm{n}=26)$ and without $M G M T$ methylation $(\mathrm{n}=26)$. Thirty-one tumors were positive for P53 expression (59\%) and 21 tumors lacked TP53 expression (Fig. 1). Tumor recurrence occurred in $44 \%$ of patients within 1 year and in $55.8 \%$ of patients after more than 1 year. Table 2 summarizes these descriptive data.

\section{Statistical analysis}

\section{Correlation of age and MGMT methylation with recurrence interval}

Glioblastomas with methylated $M G M T$ were observed more frequently in patients older than 50; however, no significant difference in recurrence interval was observed between methylated and non-methylated $M G M T$ cases in either age group $(P$-value $=0.670, P$-value $=0.667)($ Table 3$)$.
Table 1 Primers for methylation-specific polymerase chain reaction (MSP) used for testing MGMT methylation

\begin{tabular}{ll}
\hline Primer & Sequence \\
\hline MSP-MGMT-MetF & 5'-TTTCGACGTTCGTAGGTTTTCGC-3' \\
MSP-MGMT-MetR & 5'-GCACTCTTCCGAAAACGAAACG-3' \\
MSP-MGMT-UnMetF & 5'-TTTGTGTTTTGATGTTTGTAGGTTTTTGT-3' \\
MSP-MGMT-UnMetR & 5'-AACTCCACACTCTTCCAAAAACAAAACA-3' \\
\hline
\end{tabular}




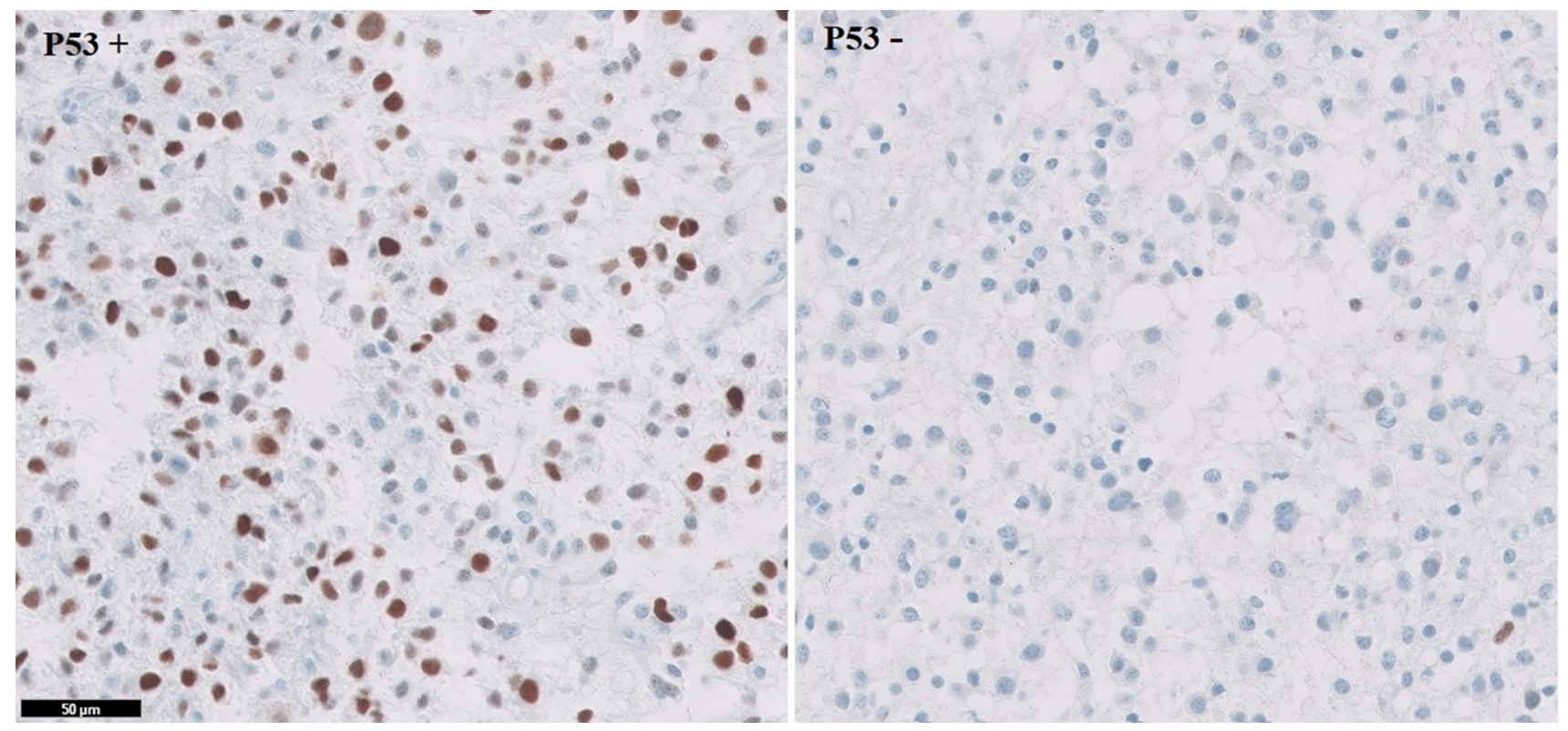

Fig. 1 TP53 expression in glioblastoma; a positive expression of a TP53 mutant; b negative expression of wild-type TP53. Scale bar $50 \mu \mathrm{m}$

Table 2 Distribution of descriptive data

\begin{tabular}{|c|c|}
\hline & $\begin{array}{l}\text { Number } \\
\text { of patients } \\
(\mathrm{n}=52)\end{array}$ \\
\hline \multicolumn{2}{|l|}{ Age at diagnosis } \\
\hline$<50$ years & $21.0(40.4 \%)$ \\
\hline$\geq 50$ years & $31.0(59.6 \%)$ \\
\hline \multicolumn{2}{|l|}{ Sex } \\
\hline Male & $31.0(59.6 \%)$ \\
\hline Female & $21.0(40.4 \%)$ \\
\hline \multicolumn{2}{|l|}{ Tumor location } \\
\hline Frontal & $20.0(38.5 \%)$ \\
\hline Temporal & $14.0(26.9 \%)$ \\
\hline Parietal & $14.0(26.9 \%)$ \\
\hline Occipital & $2.0(3.8 \%)$ \\
\hline Cerebellar & $2.0(3.8 \%)$ \\
\hline \multicolumn{2}{|l|}{$M G M T$ methylation profile } \\
\hline Non-methylated & $26.0(50.0 \%)$ \\
\hline Methylated & $26.0(50.0 \%)$ \\
\hline \multicolumn{2}{|l|}{ TP53 expression } \\
\hline Negative & $21.0(40.4 \%)$ \\
\hline Positive & $31.0(59.6 \%)$ \\
\hline \multicolumn{2}{|l|}{ Adjuvant treatment } \\
\hline None & $3.0(5.8 \%)$ \\
\hline Radiation & $4.0(7.7 \%)$ \\
\hline Radiation and chemotherapy & $45.0(86.5 \%)$ \\
\hline \multicolumn{2}{|l|}{ Recurrence interval } \\
\hline Before 1 year & $23.0(44.2 \%)$ \\
\hline After 1 year & $29.0(55.8 \%)$ \\
\hline
\end{tabular}

\section{Methylation analysis of the MGMT promoter and TP53 expression}

Fifty-two tumor samples were available for $M G M T$ promoter methylation and TP53 expression analysis. Regardless of location, $M G M T$ promoter methylation was identified in 18 cases with TP53-positive tumors (58\%), while 13 TP53-positive cases had non-methylated $M G M T$ tumors (Table 4). No significant association was found between $M G M T$ promotor methylation and TP53 expression $(P$-value $=0.158)$.

\section{TP53 expression with different treatment modalities and recurrence interval}

Although $88.9 \%$ of TP53-positive patients who received TMZ plus other chemotherapies had no tumor recurrence for over 1 year, no significant difference was found in recurrence rate with TP53-positive patients who received TMZ alone. Interestingly, around $86 \%$ of patients who were negative for TP53 expression and who received TMZ plus additional chemotherapies did not have a recurrence within 1 year $(P$-value $=0.040)$. However, a significant difference in recurrence interval was observed between TP53-positive patients versus TP53-negtive patients who received different chemotherapeutic agents (adjusted $P$-value $=0.035)($ Table 5). 
Table 3 Age distribution of glioblastoma patients with MGMT methylation status

\begin{tabular}{|c|c|c|c|c|c|c|c|}
\hline & & \multicolumn{4}{|c|}{ Recurrence interval } & \multirow[t]{3}{*}{ P-value } & \multirow[t]{3}{*}{ Adjusted P-value } \\
\hline & & \multicolumn{2}{|c|}{ Before 1 year } & \multicolumn{2}{|c|}{ After 1 year } & & \\
\hline & & $\mathrm{n}$ & $(\%)$ & $\mathrm{n}$ & $(\%)$ & & \\
\hline Age at diagnosis & $M G M T$ status & & & & & & \\
\hline \multirow{2}{*}{$<50$ Years } & Non-methylated & 7 & $(58.3)$ & 5 & $(41.7)$ & $0.670^{\mathrm{b}}$ & $0.658^{\mathrm{c}}$ \\
\hline & Methylated & 4 & $(44.4)$ & 5 & $(55.6)$ & & \\
\hline \multirow[t]{2}{*}{$\geq 50$ Years } & Non-methylated & 6 & $(42.9)$ & 8 & $(57.1)$ & $0.667^{\mathrm{a}}$ & \\
\hline & Methylated & 6 & $(35.3)$ & 11 & $(64.7)$ & & \\
\hline
\end{tabular}

No significant difference in recurrence rate was observed between patients with methylated and non-methylated MGMT in either age group

${ }^{\mathrm{a}}$ Chi-Square Test; ${ }^{\mathrm{b}}$ Fisher's Exact Test; ${ }^{\mathrm{c}}$ Mantel-Haenszel Chi-Square Test
Table 4 Relationship between MGMT promotor methylation status and TP53 expression

\begin{tabular}{lllllll}
\hline & \multicolumn{2}{l}{ MGMT status } & & P-value \\
\cline { 2 - 3 } & \multicolumn{2}{l}{ Non-methylated } & & \multicolumn{2}{l}{ Methylated } & \\
\cline { 2 - 3 } & $\mathrm{n}$ & & $\mathrm{n} \%$ & $(\%)$ & \\
\hline $\begin{array}{l}\text { TP53 expression } \\
\text { Negative }\end{array}$ & 13 & $(61.9)$ & 8 & $(38.1)$ & $0.158^{\mathrm{a}}$ \\
Positive & 13 & $(41.9)$ & & 18 & $(58.1)$ & \\
\hline
\end{tabular}

No significant association was found between $M G M T$ promotor methylation and TP53 expression

${ }^{\mathrm{a} C h i}$-Square Test

\section{Methylation analysis of the MGMT promotor in patients with different treatment modalities and recurrence intervals}

A significant difference in recurrence interval was observed among glioblastoma patients with methylated and nonemethylated MGMT, who received different chemotherapeutic protocols (adjusted $P$-value $=0.041$ ). In total, $100 \%$ of patients with non-methylated MGMT, who received TMZ plus other chemotherapies, had recurrence after 1 year $(P$-value $=0.007)$ compared with those who received TMZ
Table 5 Analysis of MGMT methylation and TP53 expression status versus recurrence rate in glioblastoma patients receiving different chemotherapies

\begin{tabular}{|c|c|c|c|c|c|c|}
\hline & \multicolumn{4}{|c|}{ Recurrence interval } & \multirow[t]{3}{*}{ P-value } & \multirow[t]{3}{*}{ Adjusted P-value } \\
\hline & \multicolumn{2}{|c|}{ Before 1 year } & \multicolumn{2}{|c|}{ After 1 year } & & \\
\hline & $\mathrm{n}$ & $(\%)$ & $\mathrm{n}$ & $(\%)$ & & \\
\hline \multicolumn{7}{|l|}{$M G M T$ status } \\
\hline \multicolumn{7}{|l|}{ Non-methylated } \\
\hline Temozolomide & 8 & $(61.5)$ & 5 & $(38.5)$ & $0.007^{\mathrm{b}}$ & $0.041^{\mathrm{c}}$ \\
\hline Temozolomide + other & 0 & $(0.0)$ & 8 & $(100.0)$ & & \\
\hline \multicolumn{7}{|l|}{ Methylated } \\
\hline Temozolomide & 6 & $(37.5)$ & 10 & $(62.5)$ & $0.667^{\mathrm{b}}$ & \\
\hline Temozolomide + other & 2 & $(25.0)$ & 6 & $(75.0)$ & & \\
\hline \multicolumn{7}{|l|}{ TP53 expression } \\
\hline \multicolumn{7}{|l|}{ Negative } \\
\hline Temozolomide & 7 & $(63.6)$ & 4 & (36.4) & $0.040^{\mathrm{a}}$ & $0.035^{\mathrm{c}}$ \\
\hline Temozolomide + other & 1 & (14.3) & 6 & $(85.7)$ & & \\
\hline \multicolumn{7}{|l|}{ Positive } \\
\hline Temozolomide & 7 & (38.9) & 11 & $(61.1)$ & $0.201^{\mathrm{b}}$ & \\
\hline Temozolomide + other & 1 & $(11.1)$ & 8 & $(88.9)$ & & \\
\hline
\end{tabular}

All patients with non-methylated $M G M T$ who received temozolomide plus other chemotherapies had delayed recurrence, after at least 1 year $(P$-value $=0.007)$ compared with patients who received TZM alone. Around $86 \%$ of patients with non-TP53 expressing tumors who received TZM plus other chemotherapies had recurrence after 1 year $(P$-value $=0.040)$

TMZ Temozolomide

${ }^{\mathrm{a}}$ Chi-Square Test; ${ }^{\mathrm{b}}$ Fisher's Exact Test; ${ }^{\mathrm{C}}$ Mantel-Haenszel Chi-square test 
alone. However, this association was not significant in the $M G M T$ methylation-positive subgroup $(P$-value $=0.667)$.

\section{Recurrence interval analysis among glioblastoma patients with methylated and non-methylated MGMT after receiving different treatment modalities}

The median recurrence time for patients with non-methylated MGMT, who received TMZ plus additional chemotherapies, was 32.6 months. The median recurrence time for patients with non-methylated MGMT but who received TMZ alone was 18.3 months. This was not significantly different from the MGMT methylation-positive group who received either TMZ alone or TMZ with or without additional chemotherapies (median recurrence $=24.4-26.4$ months) (Fig. 2a).

\section{Recurrence interval analysis among glioblastoma patients with positive and negative TP53 expression status after receiving different treatment modalities}

The median recurrence time for TP53-negative glioblastoma patients who received TMZ plus additional chemotherapies was 28.6 months. The median recurrence time for TP53negative glioblastoma patients who received TMZ alone was 12.1 months. This was not significantly different from the of TP53-positive group who received either TMZ alone or TMZ with additional chemotherapies (median recurrence $=24.4-26.4$ months) (Fig. 2b).

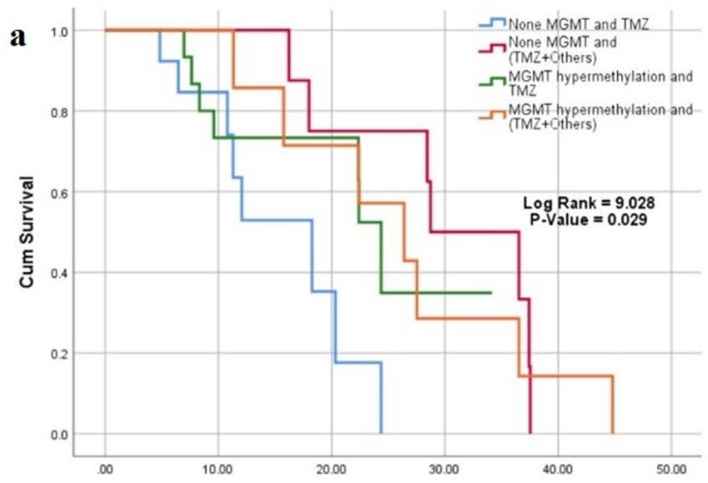

\section{Discussion}

Glioblastoma is the most common primary brain tumor in adults. The standard treatment includes surgical resection, RT and chemotherapy, mostly with the alkylating agent, TMZ. Despite this approach, outcomes are usually poor [1]. While post-operative combined treatment is the primary treatment option for newly diagnosed glioblastoma, chemotherapy resistance is still common. The efficacy of chemotherapy as an adjunct to RT is controversial. Chemotherapy with individual treatments is rarely used to treat glioblastoma after surgery but occurs in patients who refuse radiotherapy and different treatment modalities.

Several parameters have been used to predict the prognosis of glioblastoma patients. Amongst these, methylation of the $M G M T$ promoter is a biomarker for favorable outcome [2,3]. MGMT is located on chromosome band 10q26. It is a DNA repair protein that rapidly reverses alkylation (including methylation) at the $\mathrm{O} 6$ position of guanine, thereby neutralizing the cytotoxic effects of alkylating agents, such as TMZ. A lack of MGMT repair capacity contributes to the genesis and progression of human cancers because it leads to the accumulation of DNA mutations and chromosomal instability [8]. The relative expression of $M G M T$ can determine patient response to alkylating agents, and the epigenetic silencing of $M G M T$ by promoter methylation plays an active role in

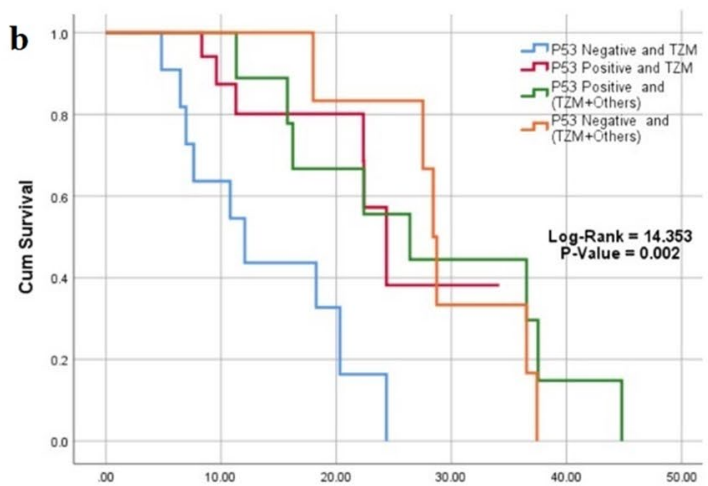

Time to Recurrence (Months)

\begin{tabular}{lcccccc}
\hline MGMT Status & Chemotherapies & N & Events & Mean & SE of Mean & Median in Months \\
\hline & & 13.00 & 8 & 15.6 & 1.98 & 18.3 \\
None methylated MGMT & TMZ & 1.00 & 7 & 29.3 & 2.71 & 32.6 \\
None methylated MGMT & TMZ Plus Other & 8.00 & 7 & 23.6 & 3.16 & 24.4 \\
Methylated MGMT & TMZ & 16.00 & 7 & 23.6 & \\
Methylated MGMT & TMZ Plus Other & 8.00 & 7 & 25.0 & 3.27 & 26.4
\end{tabular}

Fig. 2 Kaplan-Meier analysis of the recurrence interval stratified for MGMT-methylation status and TP53 expression. There was an overall significant difference in recurrence rate among glioblastoma patients receiving different chemotherapies, in regard to $M G M T$ methylation status and TP53 expression. The median recurrence rate for glioblas-

\begin{tabular}{|c|c|c|c|c|c|c|}
\hline P53 Expression & Chemotherapies & $\mathbf{N}$ & Events & Mean & SE of Mean & Median in Months \\
\hline P53 Negative & TMZ & 11.00 & 9 & 14.0 & 2.16 & 12.1 \\
\hline P53 Positive & TZM & 18.00 & 6 & 25.4 & 2.99 & 24.4 \\
\hline P53 Positive & TMZ Plus Other & 9.00 & 8 & 26.1 & 3.17 & 26.4 \\
\hline P53 Negative & TMZ Plus Other & 7.00 & 6 & 29.1 & 2.45 & 28.6 \\
\hline
\end{tabular}

tomas with non-methylated $M G M T$ that received a regime of multiple chemotherapies was 32.6 months, while the median recurrence rate for glioblastomas with TP53 expression that received a regime of multiple chemotherapies was 28.6 months 
regulating $M G M T$ expression [7]. Therefore, elevated levels of $M G M T$ activity, defined as non-methylated $M G M T$, in tumor tissue are associated with resistance to alkylating agents.

$M G M T$ promoter methylation correlates with improved tumor progression-free survival in patients treated with TMZ $[9,10]$. A meta-analysis of 34 clinical trials concluded that $M G M T$ methylation was significantly associated with better overall survival in patients with glioblastoma [11]. Hegi et al. reported that the 18-month survival rate was $62 \%$ among patients with a methylated MGMT promoter compared with only $8 \%$ in the absence of promoter methylation [5]. Some studies also showed that MGMT promoter methylation was associated with improved overall survival in patients treated with RT and TMZ but not in patients initially treated with RT alone [8]. In our study, we found that RT with TMZ and additional chemotherapies (such as lomustine and/or procarbazine and bevacizumab), particularly in unmethylated $M G M T$ tumors, was associated with a longer recurrence interval and prolonged survival rate. These observations have not been previously reported. Rapp et al. studied the impact of surgical resection, RT and concomitant TMZ in glioblastoma patients with methylated and non-methylated MGMT. They did not use TP53 expression as a factor in the prognosis [12]; however, they concluded that tumor progression-free survival and overall survival rates are strongly determined by $M G M T$ status.

Although the recurrence rate is lower and tumor progression-free survival is increased in glioblastoma patients with MGMT methylation compared with non-methylated glioblastoma, not all patients with MGMT methylation-positive glioblastoma respond to alkylating agents, and even those who respond eventually show relapse [5]. This might be because of a high rate of treatment resistance to alkylating agents in both groups. Wiewrodt et al. revealed that $M G M T$ expression is related to a change in gene expression pattern that occurs during tumor growth and progression and that it might cause therapy-related resistance [13]. Strategies to overcome $M G M T$-mediated chemoresistance are being investigated. The use of MGMT inhibitors is limited by their hematological toxicity; therefore, another strategy has been to deplete MGMT activity in tumor tissue using a dose-dense temozolomide schedule. This has been used in non-methylated $M G M T$ cases. Here, we found that the use of additional chemotherapies with TMZ treatment may have reduced the level of resistance and slowed the tumor recurrence rate to more than 1 year.

Mutations in the TP53 gene have been frequently reported in glioblastoma $[14,15]$. The immunohistochemical presence or absence of nuclear TP53 is highly significantly associated with the presence or absence of TP53 variations. Significant correlation between TP53 expression, TP53 mutations, and TP53 locus loss of heterozygosity has been shown $[14,16]$.
The regulation of $M G M T$ involves TP53, with TP53 being required for induction and also down-regulation of its basal level of expression $[4,6]$. In a recent population-based study of glioblastoma, a higher frequency of TP53 mutations (G:C > A:T transition) was found in tumors with $M G M T$ promoter methylation $(25 \%)$ than in glioblastomas without MGMT methylation (16\%) [17].

MGMT promotes methylation and TP53 mutation or P53 expression is insignificantly related. TP53 has an impact on the sensitivity of glioma cells to TMZ [6, 18]. Groenendijik et al. found no significant relationship between TP53 mutation and MGMT-promotor methylation [19]. The TP53 variant $(\mathrm{H}: \mathrm{C}>\mathrm{A})$ is commonly found in $M G M T$ methylated tumors while TP53 was not mutated in seven cases [19]. In a study conducted by Wiewrodt et al., TP53 expression was not significantly related to the level of $M G M T$ expression, although a trend for lower MGMT activity in TP53-positive tumors was observed [20]. Zawlik et al. found a significantly higher frequency of TP53 G:C > A:T transition mutations in glioblastomas with $M G M T$ promoter methylation compared with glioblastomas without $M G M T$ promoter methylation, whereas the total frequency of TP53 mutations in glioblastoma with methylated and non-methylated MGMT promoters was similar [17]. Some in vitro studies have shown that lack of TP53 expression can sensitize glioma cells to carmustine and TMZ [21, 22].

In our study, despite the $M G M T$ promotor being methylated more frequently in tumors expressing $T P 53$, no significant relationship was observed between MGMT and TP53. We also found that in glioblastomas where TP53 was not expressed, treatment with TMZ and additional chemotherapeutic agents was associated with a low rate of tumor recurrence within 1 year, which was significantly different from the rate in patients treated with TMZ alone. This shows that lack of TP53 expression may increase the sensitization of glioblastoma cells to additional chemotherapeutic agents and reduce the risk of TMZ resistance.

Finally, one limitation must be acknowledged in our study is, that the total number of cases analyzed for MGMT promoter methylation and P53 expression is relatively low. Despite this limitation and to our best knowledge, this is the first study in Saudi Arabia that correlates these molecular biomarkers with recurrence-free interval in totally resected glioblastomas, reflecting the impact of adjuvant therapies as well as the specific type of chemotherapies on patient outcome. The results are in keeping with other conclusions from similar international studies.

\section{Conclusions}

Our results show that TMZ with other chemotherapeutic agents, regardless the type and number of added drugs, produce a significantly better outcome (reduced recurrence 
interval) in glioblastoma without TP53 expression or nonmethylated MGMT promotor. This is because these glioblastomas have increased sensitivity to chemotherapies. Furthermore, TP53 mutation or expression and MGMT methylation are significant independent prognostic factors. Further studies should be performed to understand the mechanisms of glioma cell sensitivity to different chemotherapeutic agents.

Author contributions MK, idea, IRB submission, writing, study design and data analysis; NS, Statistical analysis; SB, data provider, writing and analysis; BG, study design, writing and editing; YM, data entry, tissue collection and writing; $\mathrm{AB}$, data entry and tissue collection; RS, data entry, tissue collection and writing; $\mathrm{AD}$, genetic analysis, writing and analysis; FM, tissue processing; MB, tissue processing and IHC; AS, data provider and writing; AL, data provider and IRB submission; SK, idea, data interpretation and conclusion.

Data availability The data that support the findings of this study are available from the corresponding author (MK) upon request.

\section{Compliance with ethical standards}

Conflict of interest All authors declare no conflict of interest.

Ethical approval All patients gave written informed consent. This study was approved by the National biomedical Ethics Committee of King Abdulaziz University (HA-02-J-008) which comply with the guidelines of the "System of ethics of research" prepared by the King Abdulaziz City for Science and Technology, and approved by Royal Decree No. M/59 on 24 August 2010.

Open Access This article is licensed under a Creative Commons Attribution 4.0 International License, which permits use, sharing, adaptation, distribution and reproduction in any medium or format, as long as you give appropriate credit to the original author(s) and the source, provide a link to the Creative Commons licence, and indicate if changes were made. The images or other third party material in this article are included in the article's Creative Commons licence, unless indicated otherwise in a credit line to the material. If material is not included in the article's Creative Commons licence and your intended use is not permitted by statutory regulation or exceeds the permitted use, you will need to obtain permission directly from the copyright holder. To view a copy of this licence, visit http://creativecommons.org/licenses/by/4.0/.

\section{References}

1. Waqas M, Khan I, Shamim MS (2017) Role of 5-ALA in improving extent of tumour resection in patients with glioblastoma multiforme. J Pak Med Assoc 67:1630-1632

2. Binabaj MM, Bahrami A, ShahidSales S, Joodi M, Joudi Mashhad M, Hassanian SM, Anvari K, Avan A (2018) The prognostic value of MGMT promoter methylation in glioblastoma: a metaanalysis of clinical trials. J Cell Physiol 233:378-386. https://doi. org/10.1002/jcp.25896

3. Thon N, Eigenbrod S, Grasbon-Frodl EM, Lutz J, Kreth S, Popperl G, Belka C, Kretzschmar HA, Tonn JC, Kreth FW (2011) Predominant influence of MGMT methylation in non-resectable glioblastoma after radiotherapy plus temozolomide. J Neurol Neurosurg
Psychiatry 82:441-446. https://doi.org/10.1136/jnnp.2010.21459 3

4. Grombacher T, Eichhorn U, Kaina B (1998) p53 is involved in regulation of the DNA repair gene O6-methylguanine-DNA methyltransferase (MGMT) by DNA damaging agents. Oncogene 17:845-851. https://doi.org/10.1038/sj.onc.1202000

5. Hegi ME, Liu L, Herman JG, Stupp R, Wick W, Weller M, Mehta MP, Gilbert MR (2008) Correlation of O6-methylguanine methyltransferase (MGMT) promoter methylation with clinical outcomes in glioblastoma and clinical strategies to modulate MGMT activity. J Clin Oncol 26:4189-4199. https://doi.org/10.1200/ JCO.2007.11.5964

6. Grombacher T, Mitra S, Kaina B (1996) Induction of the alkyltransferase (MGMT) gene by DNA damaging agents and the glucocorticoid dexamethasone and comparison with the response of base excision repair genes. Carcinogenesis 17:2329-2336

7. Esteller M, Hamilton SR, Burger PC et al (1999) Inactivation of the DNA repair gene O6-methylguanine-DNA methyltransferase by promoter hypermethylation is a common event in primary human neoplasia. Cancer Res 59:793-797

8. Gerson SL (2002) Clinical relevance of MGMT in the treatment of cancer. J Clin Oncol 20:2388-2399

9. Esteller M, Hamilton SR, Burger PC, Baylin SB, Herman JG (1999) Inactivation of the DNA repair gene O6-methylguanineDNA methyltransferase by promoter hypermethylation is a common event in primary human neoplasia. Cancer Res 59:793-797

10. Hegi ME, Diserens AC, Gorlia T, Hamou MF, de Tribolet N, Weller M, Kros JM, Hainfellner JA, Mason W, Mariani L, Bromberg JE, Hau P, Mirimanoff RO, Cairncross JG, Janzer RC, Stupp R (2005) MGMT gene silencing and benefit from temozolomide in glioblastoma. N Engl J Med 352:997-1003. https://doi. org/10.1056/NEJMoa043331

11. Beranek DT (1990) Distribution of methyl and ethyl adducts following alkylation with monofunctional alkylating agents. Mutation Res 231:11-30

12. Zawlik I, Vaccarella S, Kita D, Mittelbronn M, Franceschi S, Ohgaki H (2008) Promoter methylation and polymorphisms of the MGMT gene in glioblastomas: a population-based study. Neuroepidemiology 32:21-29

13. Wiewrodt D, Nagel G, Dreimüller N, Hundsberger T, Perneczky A, Kaina B (2008) MGMT in primary and recurrent human glioblastomas after radiation and chemotherapy and comparison with p53 status and clinical outcome. Int J Cancer 122(6):1391-1399. https://doi.org/10.1002/ijc.23219

14. Watanabe K, Sato K, Biernat W, Tachibana O, von Ammon K, Ogata N, Yonekawa Y, Kleihues P, Ohgaki H (1997) Incidence and timing of p53 mutations during astrocytoma progression in patients with multiple biopsies. Clin Cancer Res 3:523-530

15. Reifenberger J, Ring GU, Gies U, Cobbers L, Oberstrass J, An HX, Niederacher D, Wechsler W, Reifenberger G (1996) Analysis of p53 mutation and epidermal growth factor receptor amplification in recurrent gliomas with malignant progression. J Neuropathol Exp Neurol 55:822-831. https://doi.org/10.1097/00005 072-199607000-00007

16. Wiewrodt D, Nagel G, Dreimüller N, Hundsberger T, Perneczky A, Kaina B (2008) MGMT in primary and recurrent human glioblastomas after radiation and chemotherapy and comparison with P53 status and clinical outcome. Int J Cancer 122:1391-1399

17. Friedman HS, McLendon RE, Kerby T, Dugan M, Bigner SH, Henry AJ, Ashley DM, Krischer J, Lovell S, Rasheed K, Marchev F, Seman AJ, Cokgor I, Rich J, Stewart E, Colvin OM, Provenzale JM, Bigner DD, Haglund MM, Friedman AH, Modrich PL (1998) DNA mismatch repair and O6-alkylguanine-DNA alkyltransferase analysis and response to Temodal in newly diagnosed malignant glioma. J Clin Oncol 16:3851-3857. https://doi.org/10.1200/ JCO.1998.16.12.3851 
18. Roos WP, Batista LFZ, Naumann S, Wick W, Weller M, Menck CFM, Kaina B (2007) Apoptosis in malignant glioma cells triggered by the temozolomide-induced DNA lesion O6-methylguanine. Oncogene 26:186-197

19. Rapp M, Geoppert M, Falsberg J, Steiger HJ, Sabel M (2013) The impact of sequencial versus combined radiochemotheray with temozolomide, resection, and MMT promotor hypermethylation on survival of patients with primary glioblastoma-a single centre retrospective study. Br J Neurosurg 27(4):430-435

20. Groenendijk FH, Taal W, Dubbink HJ, Haarloo CR, Kouwenhoven MC, van den Bent MJ, Kros JM, Dinjens WN (2001) MGMT promotor methylation is a frequent, early, and consistent event in astrocytoma progression, and not correlated with TP53 mutation. J Neuroncol 101:405-417
21. Ständer M, Peraud A, Leroch B, Kreth FW (2004) Prognostic impact of TP53 mutation status for adult patients with supratentorial World Health Organization Grade II astrocytoma or oligoastrocytoma: a long-term analysis. Cancer 101:1028-1035

22. Li S, Zhang W, Chen B, Jiang T, Wang Z (2010) Prognostic and Predictive value of P53 in low MGMT expression glioblastoma treated with surgery, radiation, and adjuvant temozolomide chemotherapy. Neurol Res 7:690-694

Publisher's Note Springer Nature remains neutral with regard to jurisdictional claims in published maps and institutional affiliations. 